\title{
The CMS Outer Tracker Upgrade for the High Luminosity LHC
}

\author{
Jelena Luetić* ${ }^{*}$ \\ Université Libre de Bruxelles (BE) \\ E-mail: jelena.luetic@cern.ch
}

The era of the High Luminosity Large Hadron Collider will pose unprecedented challenges for detector design and operation. The planned luminosity of the upgraded machine is $5 \times 10^{34} \mathrm{~cm}^{-2} \mathrm{~s}^{-1}$, reaching an integrated luminosity of more than $3000 \mathrm{fb}^{-1}$ by the end of 2037 . The CMS Tracker detector will have to be replaced in order to fully exploit the delivered luminosity and cope with the demanding operating conditions. The new detector will provide robust tracking as well as input for the first level trigger. This report is focusing on the replacement of the CMS Outer Tracker system, describing the new layout and technological choices together with some highlights of research and development activities.

The 26th International Workshop on Vertex Detectors

10-15 September, 2017

Las Caldas, Asturias, Spain

\footnotetext{
*Speaker.

${ }^{\dagger}$ on behalf of the CMS collaboration
} 


\section{Introduction}

The high luminosity Large Hadron Collider (HL-LHC) will begin a new era in the life of the CERN LHC. The accelerator upgrade will be installed during the long shutdown period in 2024 and 2025. The main goal is to be able to deliver an increased instantaneous luminosity of $5 \times 10^{34}$ $\mathrm{cm}^{-2} \mathrm{~s}^{-1}$, with a peak value reaching up to $7.5 \times 10^{34} \mathrm{~cm}^{-2} \mathrm{~s}^{-1}$. Such conditions pose unprecedented requirements on the detectors as the number of pile-up events increases to 140 events $^{1}$. Thus, good spatial and time resolution become increasingly important in order to distinguish between different primary events. During the lifetime of the HL-LHC, an integrated luminosity of $3000-4000 \mathrm{fb}^{-1}$ will be collected, thus allowing studies of rare and suppressed events.

Due to the expected CMS Tracker detector deterioration in performance, it will have to be fully replaced before the HL-LHC operation. The new detector will have improved radiation tolerance to withstand radiation levels up to $10^{16} \mathrm{n}_{\mathrm{eq}} / \mathrm{cm}^{2}$. In order to distinguish between the different primary interactions and maintain high tracking efficiency, an increase in the detector granularity is planned. Additional improvement of the tracking efficiency arises from the smaller material budget within the Tracker volume. Selection of good events at the first trigger level will be enhanced by the Tracker information, ensuring the rejection of tracks with low momentum. A complete overview of the current state of plans and developments for the CMS Tracker Phase-2 Upgrade can be found in [1].

\section{Outer Tracker geometry}

The new CMS Tracker detector comprises two major parts. The Inner Tracker (IT) placed at the lower radii around the collision point will be built using silicon pixel technology. The Outer Tracker (OT) located further away from the collision point, will be constructed with the combination of silicon pixel and silicon strip technology. The baseline design for Outer Tracker foresees two kinds of modules, $2 \mathrm{~S}$ modules with two closely spaced parallel strip sensors and PS modules that are constructed with one macro-pixel and one strip sensor. The Outer Tracker will consist of six cylindrical layers in the central barrel region of $|z|<1200 \mathrm{~mm}$ and five double-discs on each side, in the region of $1200<|z|<2700 \mathrm{~mm}$ (Figure 1). Particles emerging from the collision region $(|z|<70 \mathrm{~mm}$ ) will cross all six modules in the pseudorapidity range of up to $|\eta|<2.4$, apart from a small region where the barrel and endcap regions connect, thus ensuring hermeticity. Part of the barrel region is realized with the modules tilted with respect to the $\mathrm{z}$ axis, as it was shown in the simulations that better performance can be achieved with reduced cost and amount of material [1].

\section{Outer Tracker module design}

The upgrade of the Outer Tracker is closely connected to the upgrade of the CMS Trigger system [2]. With increasing pile-up, the trigger system will have to handle higher output rates of interesting events and improve the discriminatory power of event selection. Incorporating more sources of event information into the trigger system and increasing the time available to make a

\footnotetext{
${ }^{1}$ In the most challenging performance scenario, the number of pile-up events could reach 200.
} 


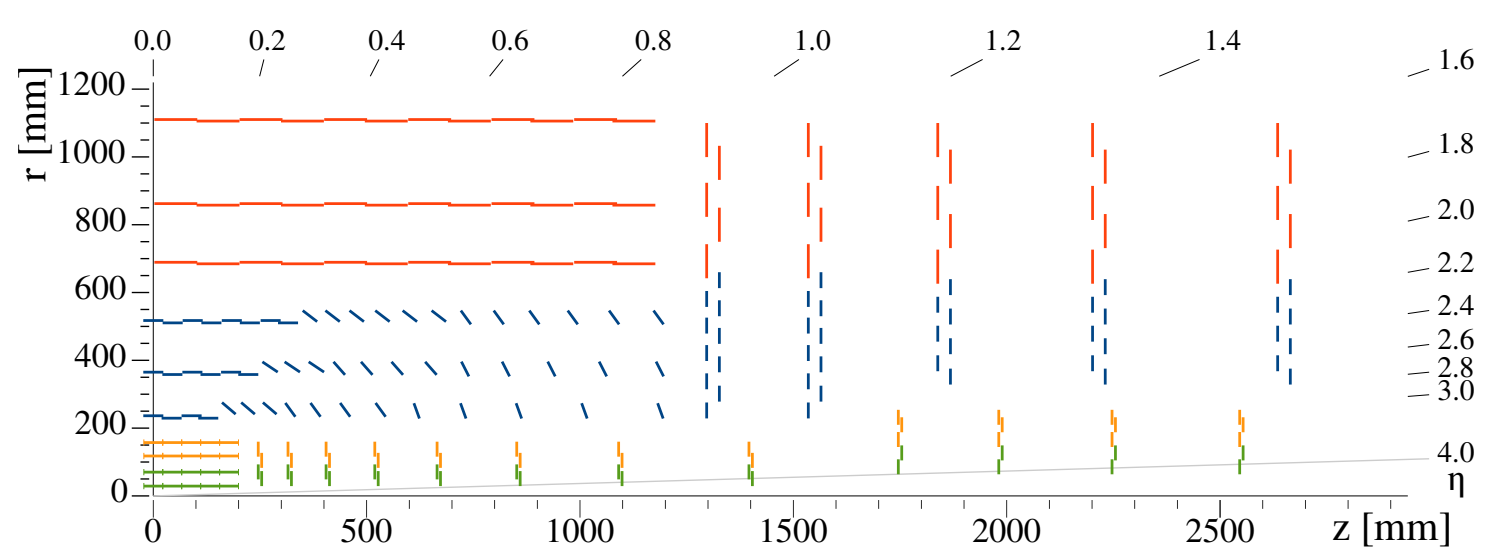

Figure 1: Tracker geometry

decision will lead to an improved discriminating power. Outer Tracker modules will provide a rough information about the track transverse momentum $\left(p_{\mathrm{T}}\right)$ to the first level of the CMS Trigger system (L1 trigger), allowing for better transverse momentum resolution of various objects at L1 and mitigation of pile-up effects. There is a plan to enhance the first level trigger rate from present $100 \mathrm{kHz}$ to $750 \mathrm{kHz}$. The amount of time available for data transfer and running of the L1 trigger algorithms corresponds to the trigger latency and will increase to $12.5 \mu$ s from the present value of $3.2 \mu \mathrm{s}$. The front-end electronics and the L1 trigger track reconstruction need to comply with these new requirements.

\section{1 $p_{\mathrm{T}}$ module concept}

The main idea behind the Outer Tracker module design is implementing the trigger at the tracker level. Such a novel concept implies that the modules have to provide data about the event at the bunch crossing rate. The front-end electronics will be responsible for the data volume reduction by simple and fast reconstruction, roughly determining the incoming particle $p_{\mathrm{T}}$. The modules are then capable of rejecting all the data from particles below a certain $p_{\mathrm{T}}$ threshold. The design of these so-called $p_{\mathrm{T}}$ modules exploits the bending of the particle trajectory inside the $3.8 \mathrm{~T}$ magnetic field of the CMS detector as a function of the particle $p_{\mathrm{T}}$. The transverse momentum is determined by making two closely spaced measurements with two silicon sensors read out by the same set of ASICs. The ASICs will make correlations between the hit pairs (later referred as "stubs"), selecting the ones that are compatible with the particles above the $p_{\mathrm{T}}$ threshold. Simulations show that a $p_{\mathrm{T}}$ cut of $2 \mathrm{GeV}$, reduces the data by a factor ten, making the stub information transfer at the bunch crossing frequency a viable option. The $p_{\mathrm{T}}$ threshold is selected by tuning the readout chip setting corresponding to the search window. Moreover, in order to ensure good track selection performance and minimize the contributions from random coincidences, modules with different spacings between the sensors will be built, with distances ranging between $1.6 \mathrm{~mm}$ and $4 \mathrm{~mm}$. Stub coordinates are then transmitted to the track finding system that combines the data from all modules, and sends it to the CMS L1 trigger system. Full event information is stored in the pipeline on the front-end readout ASIC, waiting for the L1 trigger to accept the signal when it is transmitted to the CMS data acquisition system. 


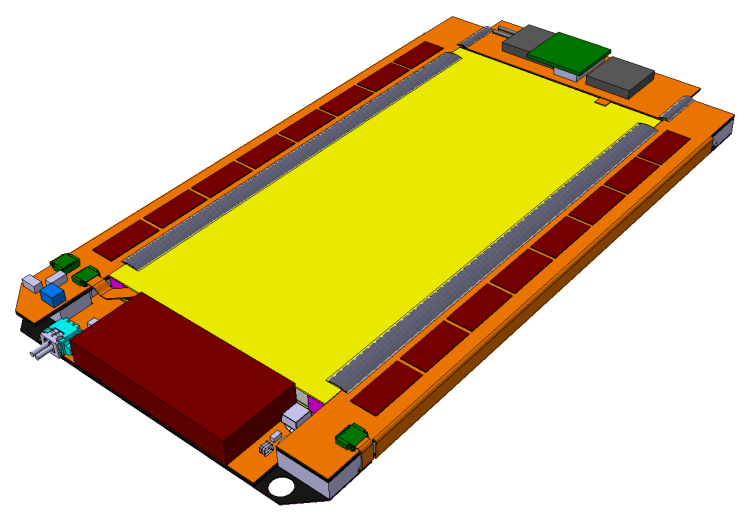

(a) PS Module

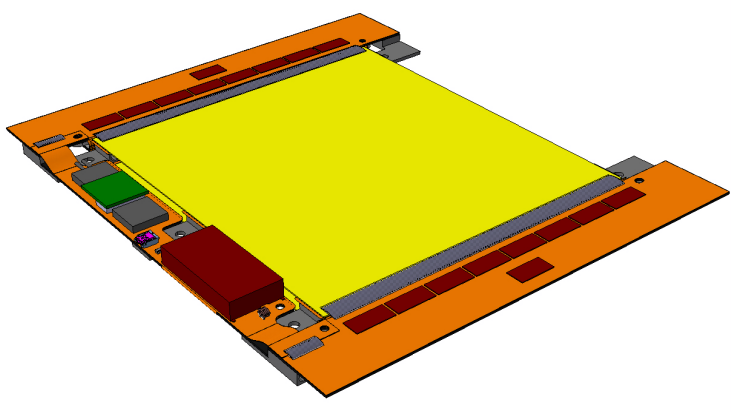

(b) $2 \mathrm{~S}$ Module

Figure 2: Two varieties of CMS Outer tracker $p_{\mathrm{T}}$ modules

\subsection{PS and 2S modules}

Two versions of $p_{\mathrm{T}}$ modules are envisioned for the Outer Tracker upgrade. Modules with two parallel strip sensors ( $2 \mathrm{~S}$ modules) will be placed in the outer layers of the tracker volume, while in the inner layers, there will be modules with one strip and one macro-pixel sensor (PS modules). Both variants can be seen in Figure 2. Sensors used for module construction will be of n-on-p type with an active thickness of around $200 \mu \mathrm{m}$. The surface of $2 \mathrm{~S}$ module sensors is $10 \times 10 \mathrm{~cm}^{2}$, with strip length of $5 \mathrm{~cm}$ and pitch of $90 \mu \mathrm{m}$. The design of the strip sensor of the PS module is similar to the one of $2 \mathrm{~S}$ with an area of $5 \times 10 \mathrm{~cm}^{2}$ and strip length of $2.35 \mathrm{~cm}$. The PS strip sensor pitch is $100 \mu \mathrm{m}$. The macro-pixel sensor has $1467 \mu \mathrm{m}$ long pixels with pitch of $100 \mu \mathrm{m}$ [3]. The spacing between the sensors is achieved by gluing them onto aluminium/carbon fibre spacers (Al-CF) that provide mechanical stability and efficient heat removal. Strip sensors will be wire bonded to the front-end hybrids for readout. The hybrid consists of a single Kapton flex circuit board that is folded and laminated onto stiffeners of the same thickness as the Al-CF spacers between the sensors. 2S hybrids carry eight CMS Binary Chips (CBCs) [4] connected to the top and bottom sensor and a Concentrator Integrated Circuit (CIC) [5], which buffers, aggregates and sparsifies data from the $\mathrm{CBC}$ chips sending it further down to the readout link. CIC is also responsible for delivering clock, trigger and control signals to the CBC chips. The PS front-end hybrid reads out strip sensors with Short-Strip ASICs (SSAs) [6] and CIC chip. The pixel sensor is bump-bonded to the Macro-pixel ASIC (MPA), responsible for reading out pixel data and correlating it to the data from the SSAs [7]. All chips have a binary readout. Electronics for powering front-end chips and optical data readout are placed on the service hybrids. $2 S$ modules contain one service hybrid on one end of the module. In the PS module, services are split in two hybrids, on opposite ends of the module, with one side housing powering functionalities while the other performs control and readout operations. Electronics components associated with the service hybrid are part of the common CERN developments for multiple upgrade projects.

These include the following:

- $D C$-DC converter - generates voltages for front-end electronics and optoelectronics, 
- HV connector - provides bias voltage for the sensors,

- LpGBT chip - serializes/deserializes data to/from back-end, controls front-end ASICs and distributes clock and trigger,

- VTRx+ optoelectronic transceiver - converts data signals to optical/electric.

Service hybrids are connected to the back-end electronics transferring stub data to the dedicated track trigger boards and L1 triggered data to the data acquisition system.

\subsection{Track trigger}

The design of the Outer Tracker is largely driven by the need to process large amount of L1 trigger data. The main goal of the system is to reduce the amount of tracks with low transverse momentum $\left(p_{\mathrm{T}}<2 \mathrm{GeV}\right)$, which is done by correlating two closely spaced measurements taken with modules described earlier in this section. This procedure allows formation and selection of stubs consistent with the chosen transverse momentum cut with high efficiency. Good performance throughout the detector is ensured by a programmable correlation window and offset between two sensors. Data reduction in the scale of 10 to 100 times is expected, depending on the track location. In the CMS experiment three different approaches to L1 tracking have been investigated:

- AM FPGA: based on FPGA and specially designed associative memory ASICs, which rely on pattern recognition of previously loaded patterns inside the ASIC, with final selection done by the FPGA,

- Hough transform: two step approach with coarse stub selection done using Hough transform [8] and final track selection with Kalman filter [9],

- Tracklet: based on road-search algorithm, where stubs in neighbouring layers form a seed, which is used in a linearised $\chi^{2}$ fit to obtain final track parameters.

For each of these approaches, a dedicated demonstration system has been constructed. Demonstrators have shown that all three approaches would be able to achieve the necessary performance in terms of delivering tracks within the imposed timing constraints using simulated data. Future developments are aimed at combining the three approaches for building a final system.

\section{Prototyping and testing}

Several prototype modules have been built and their performance and stability have been tested. Measurements have been performed to ensure a good performance in the conditions as close as possible to the real operating conditions and to estimate the possible effects of external conditions such as humidity and temperature. In order to evaluate the module response and irradiation effects, several beam tests have been conducted at CERN, DESY and FNAL. 


\subsection{CBC2 mini module}

The performance of the CBC chip has been tested by building two CBC2 mini-modules. Such a module comprises a hybrid with two early prototype versions of the $\mathrm{CBC}$ chip with most of the stub finding logic implemented [12]. The hybrid is wire-bonded to two silicon strip sensors with 254 strips of $5 \mathrm{~cm}$ length. Several mini-modules were built and their performance tested in beam tests as well as in various system tests, assessing the stability of the long term operation in nominal conditions. A separate long-term operational stability test was performed with one CBC2 minimodule. The module was operated at $-30^{\circ} \mathrm{C}$ for a week in order to measure the effects of the long term cold operation on the noise levels. The noise was measured as a standard deviation of the S-curve, showing that the noise level as a function of time was stable around what corresponds roughly to 1000 electrons, which is well below the signal level for a MIP particle (Figure 3). The fluctuations in the measurement correspond to small changes in the environmental conditions.

\subsubsection{Beam test}

A $2 \mathrm{~S}$ mini-module beam test was carried out at the CERN H6B beam line using $120 \mathrm{GeV}$ pions. Reference tracks were reconstructed with the AIDA telescope [10] and matched to hits in the trigger plane using the ATLAS FE-I4 chip [11]. A comparison between stub efficiencies of irradiated and nonirradiated mini-modules was performed with the results shown in Figure 4. The irradiated mini-module was exposed to a fluence of $6 \times 10^{14} \mathrm{n}_{\mathrm{eq}} / \mathrm{cm}^{2}$ corresponding to twice the maximal fluence expected in the CMS Outer Tracker. The stub reconstruction efficiency is defined as the fraction of events with one reconstructed track that contains at least one reconstructed stub. The matching between track and stub is performed within $4 \sigma$ of the spatial resolution of the sensor. For the non-irradiated module, the sharp rise in the efficiency and the plateau at $99 \%$ prove that this module can efficiently select tracks above a certain threshold. For the irradiated module, the plateau is reached at $95 \%$ of the efficiency. The $p_{\mathrm{T}}$ resolution in both cases is around $5 \%$.

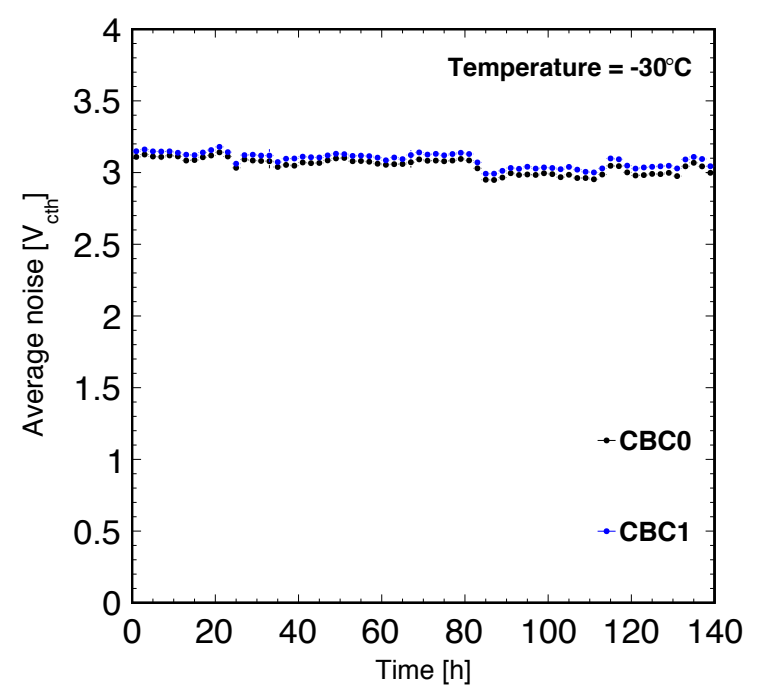

Figure 3: Mini-module long term cold operation. 


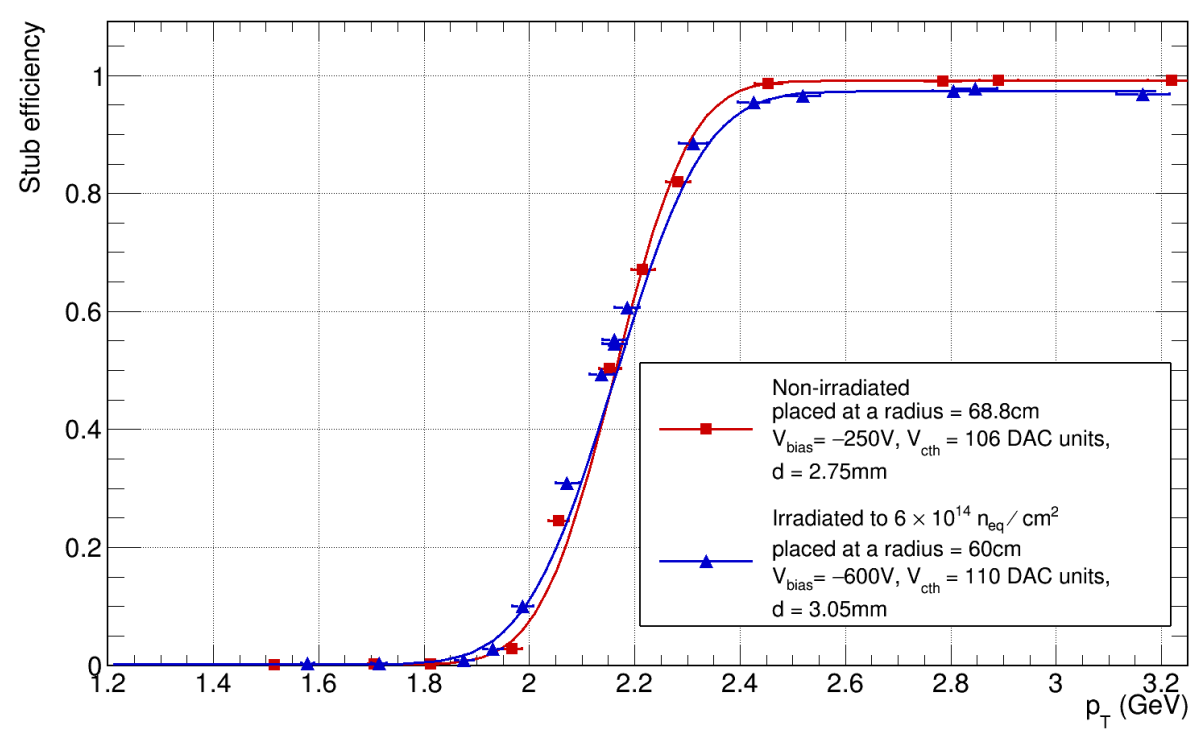

Figure 4: Mini-module stub reconstruction efficiency as a function of particle transverse momentum. The red curve shows measurements for a non-irradiated mini-module, while the blue curve shows measurements done for an irradiated mini-module.

\subsection{CBC2 full module}

This version of prototype module was built with two full $2 \mathrm{~S}$ sensors wire-bonded to two Kapton flex hybrids including eight $\mathrm{CBC} 2$ chips each. In the absence of the concentrator chip and the service hybrid, all differential lines from the chips were read out directly from the connector and dedicated low and high voltage connections were made. As a part of the module characterization, the noise level was measured at room temperature for two different modules. Studies based on the internal calibration pulse of the readout $\mathrm{CBC} 2$ chip show that the measured noise corresponds to around 1000 electrons and is relatively uniform across all tested hybrids. Another test was performed on a full module powered with and without the prototype service hybrid. This prototype hybrid contains a DC-DC converter to supply necessary voltages to the chips. The goal of the test was to see if the close proximity of a DC-DC converter would introduce additional noise into the system. The results can be seen in Figure 5. The noise levels are comparable across the module with only two chips closest to the converter having significantly different noise levels. These are, however, still well below the signal levels.

Testing the full $\mathrm{CBC} 2$ module in the test beam was carried out at the same H6B test beam facility at CERN used also for testing the $\mathrm{CBC} 2$ mini-module. The module was mounted on a translator stage and scanned with the beam to measure the stub reconstruction efficiency per strip. The average efficiency was found to be around $97 \%$ and is shown in Figure 6. The efficiency is lower than the one measured for mini-modules and it arises from the contamination with the events where the synchronization between the telescope and the module was lost. 


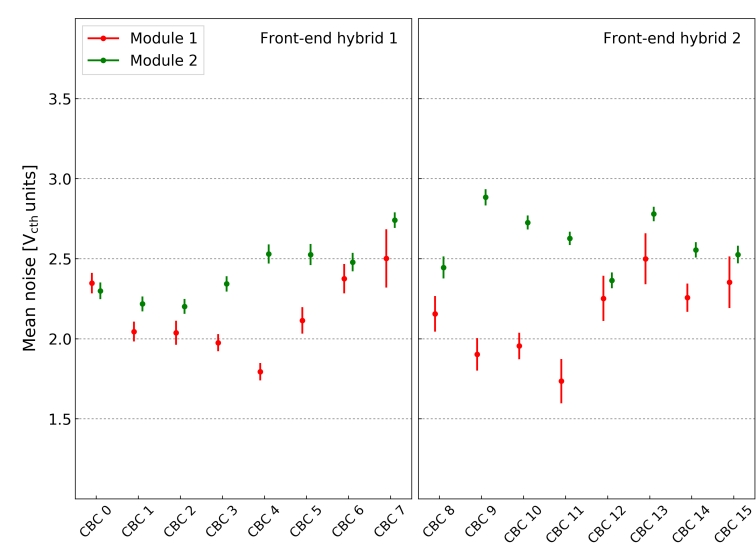

(a) Noise measurements for two full modules

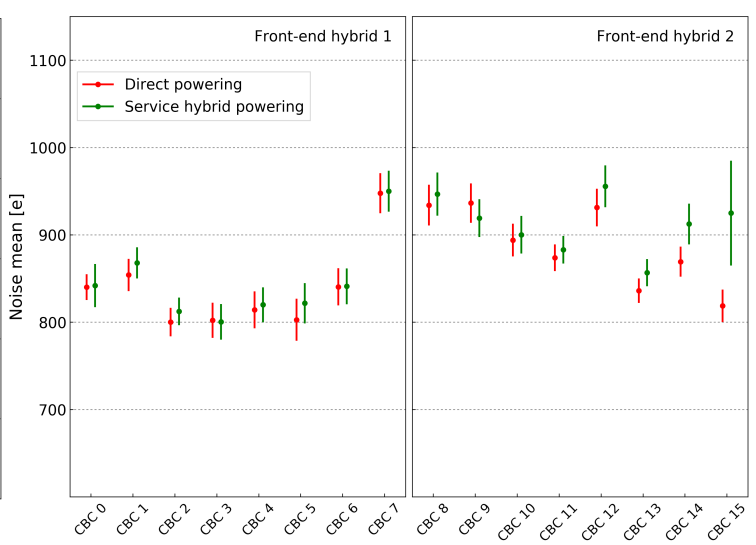

(b) Noise measurement with prototype service hybrid housing a DC-DC converter

Figure 5: Full 2S module system tests

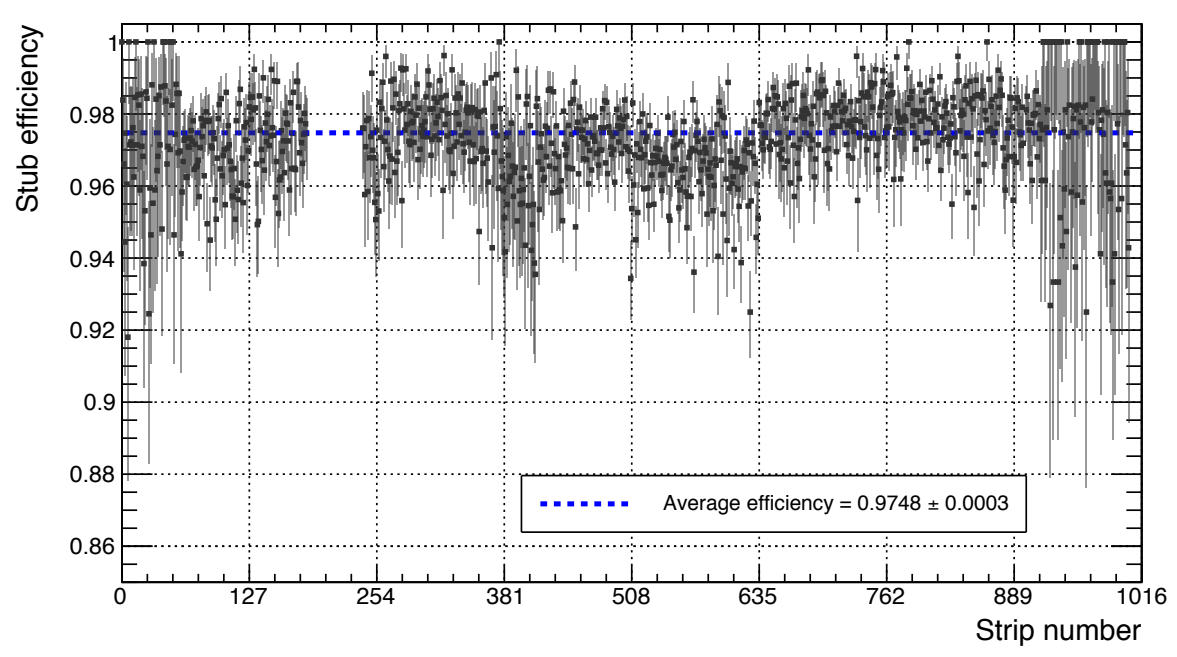

Figure 6: Full 2S module stub reconstruction efficiency measured with test beam

\subsection{MaPSA-light}

A prototype version of an MPA chip with 48 channels (MPA-light) was used to construct an assembly of six chips bump bonded to a bare module, called macro-pixel sub-assembly (MaPSA). The assembly was also used for the optimization of the bump bonding process. In order to asses the stub-finding capabilities of the MPA chip, two MaPSAs were stacked together with a few millimetre gap, creating a PS micro-module. One assembly was set to strip emulation mode, in which the MPA readout chip can output strip-detector like signals, while the other was responsible for stub finding logic.

The MaPSA-light assembly was tested in detail at a FNAL test beam facility providing $120 \mathrm{GeV}$ protons. The hit reconstruction efficiency as a function of the phase difference of the particle ar- 
rival time and the MPA clock was measured to asses the response of the MaPSA-light assembly. Due to different frequencies of the beam clock and the MPA clock jitter was introduced into the measurement of the particle arrival time. The jitter-corrected efficiency distribution is obtained by fitting and unfolding the efficiency spectrum. The result is shown in Figure 7. The hit efficiency is close to $100 \%$ and the dependence of the efficiency on the arrival time is consistent with the specifications.

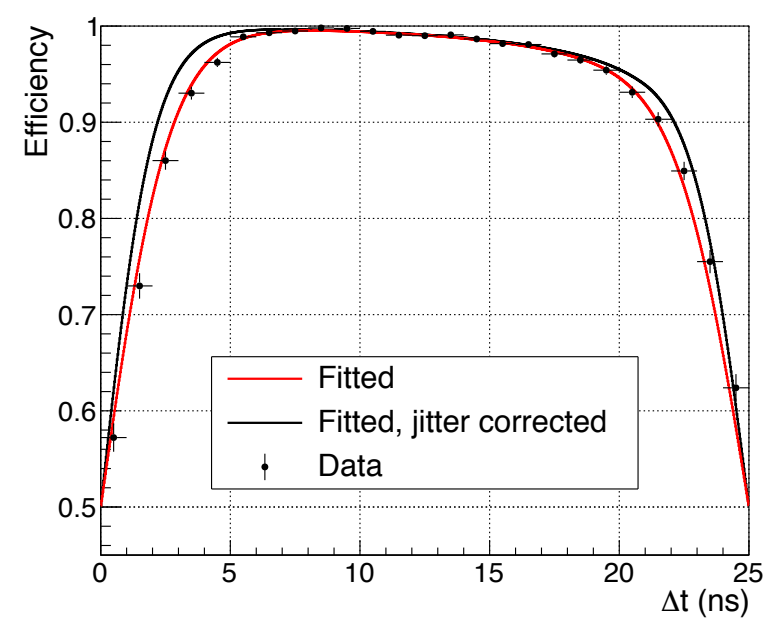

Figure 7: MaPSA test beam results

\section{Conclusions}

The upcoming HL-LHC upgrade poses numerous challenges for the experiments for maintaining an excellent physics performance. In order to operate in the high luminosity conditions, the CMS Outer Tracker will have to undergo a full replacement. New modules that are able to perform filtering based on the transverse momentum of the incoming particle and provide information to the L1 triggering system are currently being designed. A number of prototypes has already been built and they are showing promising results even after being exposed to high radiation fluences.

\section{References}

[1] K. Klein, The Phase-2 Upgrade of the CMS Tracker, CERN-LHCC-2017-009, CMS-TDR-014, CERN, Geneva, June 2017.

[2] CMS Collaboration, Technical Proposal for the Phase-II Upgrade of the CMS Detector, CERN-LHCC-2015-010, LHCC-P-008, CMS-TDR-15-02 (2015).

[3] A. Dierlamm for the CMS Collaboration, Characterisation of silicon sensor materials and designs for the CMS Tracker Upgrade, PoS (Vertex2012) 016 (2013).

[4] D. Braga, Development of the Readout Electronics for the High Luminosity Upgrade of the CMS Outer Strip Tracker, PhD thesis, Imperial College London, January 2016.

http: //hdl. handle.net/10044/1/33725. 
[5] D. Braga et al., I/O data formats for the Concentrator Integrated Circuit, 13.6.2016. https: / /espace.cern.ch/Tracker-Upgrade/Electronics/CIC/ Shared\%20Documents/Forms/Allitems.aspx.

[6] A. Caratelli et al., Short Strip ASIC Specifications Document, September 2016. https://espace.cern. ch/CMS-MPA/SitePages/Documents.aspx.

[7] D. Ceresa et al., Macro Pixel ASIC (MPA): the readout ASIC for the pixel-strip (PS) module of the CMS outer tracker at HL-LHC, 2014 JINST 9 C11012, doi:10.1088/1748-0221/9/11/C11012.

[8] P. V. C. Hough, Method and means for recognizing complex patterns, US Patent 3,069,654, December 1962.

[9] R.Fruhwirth, Application of Kalman filtering to track and vertex fitting, Nucl. Instrum. Meth. A262 (1987) 444, doi:10.1016/0168-9002(87) 90887-4.

[10] I. Rubinskiy, An EUDET/AIDA Pixel Beam Telescope for Detector Development, Physics Procedia 37 (2012) 923, doi:10.1016/j.phpro.2012.02.434.

[11] M. Barbero, The FE-I4 Pixel Readout Chip and the IBL Module, Technical Report, ATL-UPGRADE-PROC-2012-001, CERN, Geneva, 2012.

[12] G. Hall et al., CBC2: A CMS microstrip readout ASIC with logic for track-trigger modules at $H L-L H C$, Nuclear Instruments and Methods in Physics Research Section A, Volume 765, 2014, Pages 214-218, doi:10.1016/j.nima.2014.04.056. 